Supporting Information

\title{
High-Capacity and Rapid Removal of Refractory NOM Using Nanoscale Anion Exchange Resin
}

Billy R. Johnson, ${ }^{a}$ Tim B. Eldred, ${ }^{a}$ Andy T. Nguyen, ${ }^{a}$ William M. Payne, ${ }^{a}$ Emily E. Schmidt, ${ }^{a}$ Amir Y. Alansari, ${ }^{b}$ James E. Amburgey, ${ }^{b}$ and Jordan C. Poler ${ }^{a *}$

${ }^{a}$ Department of Chemistry, University of North Carolina at Charlotte, Charlotte, NC

${ }^{b}$ Department of Civil and Environmental Engineering, University of North Carolina at Charlotte, Charlotte, NC

$\mathrm{a}^{*}$ Corresponding Author: Department of Chemistry, University of North Carolina at Charlotte, Charlotte, NC Fax: (704) 687-0960; Tel: (704) 687-8289; E-mail: jcpoler@uncc.edu 


\section{LIST OF ABBREVIATIONS}

\begin{tabular}{|c|c|}
\hline $\mathrm{AA}$ & L-ascorbic acid \\
\hline AER & anion exchange resin \\
\hline ARGET & activators regenerated by electron transfer \\
\hline ATR & attenuated total reflectance \\
\hline ATRA & atom transfer radical addition \\
\hline ATRP & atom transfer radical polymerization \\
\hline $\mathrm{BB}$ & benzyl bromide \\
\hline $\mathrm{BDE}$ & bond dissociation energy \\
\hline bipy & 2,2'-bipyridine \\
\hline CRP & controlled radical polymerization \\
\hline DBP & disinfection byproduct \\
\hline DLS & dynamic light scattering \\
\hline $\mathrm{DMF}$ & N,N-dimethylformamide \\
\hline DOC & dissolved organic carbon \\
\hline DP & degree of polymerization \\
\hline EPM & electrophoretic mobility \\
\hline FTIR & Fourier transform infrared spectroscopy \\
\hline GAC & granular activated carbon \\
\hline HAA & haloacetic acid \\
\hline HEBiB & 2-hydoxyethyl 2-bromoisobutyrate \\
\hline HiPCO & high pressure carbon monoxide \\
\hline IEX & ion exchange \\
\hline $\mathrm{mg}-\mathrm{C} / \mathrm{L}$ & milligrams of carbon per liter of solution \\
\hline $\mathrm{MIEX}^{(\mathbb{R})}$ & magnetic ion exchange \\
\hline MS & mass spectrometry \\
\hline
\end{tabular}




$\begin{array}{ll}\text { MW } & \text { molecular weight } \\ \text { MWCNT } & \text { multi-walled carbon nanotube } \\ \text { NaFL } & \text { sodium fluorescein } \\ \text { NMR } & \text { nuclear magnetic resonance } \\ \text { NOM } & \text { natural organic matter } \\ \text { PAC } & \text { powdered activated carbon } \\ \text { RBM } & \text { radial breathing modes } \\ \text { SBA } & \text { strong base anion exchange resin } \\ \text { SEM } & \text { scanning electron microscopy } \\ \text { SSA } & \text { specific surface area } \\ \text { SUVA } & \text { specific UV absorbance } \\ \text { SWCNT } & \text { single-walled carbon nanotube } \\ \text { THM } & \text { trihalomethane } \\ \text { TOC } & \text { total organic carbon } \\ \text { TPMA } & \text { tris(2-pyridylmethyl)amine } \\ \text { USEPA } & \text { United States Environmental Protection Agency } \\ \text { vbTMAC } & \text { vinylbenzyl trimethylammonium chloride } \\ \text { WBA } & \text { weak base anion exchange resin } \\ & \end{array}$




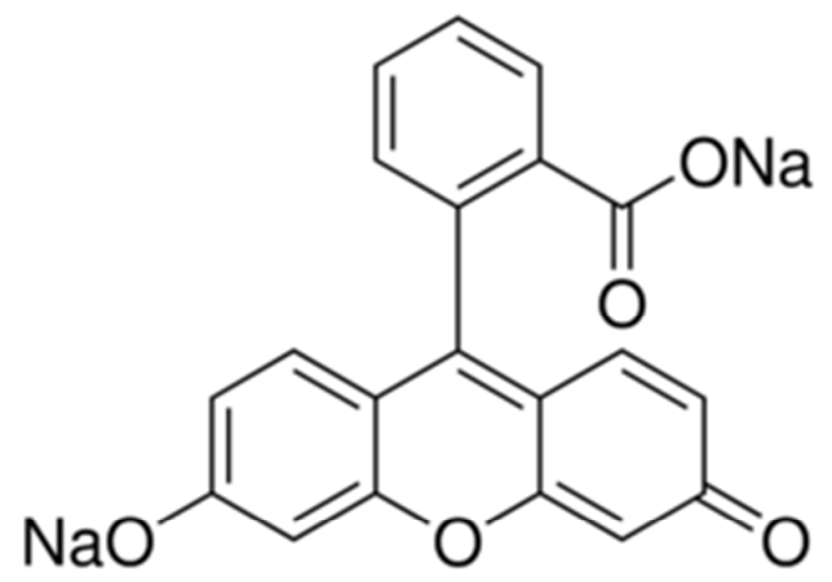

Fig. S1. Sodium fluorescein was chosen as a surrogate molecule for NOM due to structural similarities to fulvic acids and strong absorbance in the visible region. A large majority of NOM has either carboxylate or phenolate groups, or both, leaving them as negatively charged species in water. 
Table S1. Coagulation data for low MW surrogate, sodium fluorescein. About $5 \%$ of the surrogate could be removed via coagulation. Proving the difficulty found in removing these types of low MW compounds from drinking water sources using coagulation.

\begin{tabular}{|c|c|c|c|c|c|}
\hline \multirow{2}{*}{ Sample } & \multicolumn{2}{|c|}{ UV254 } & \multicolumn{2}{c|}{ DOC [mg-c/L] } & \multirow{2}{*}{ SUVA } \\
& Avg. (n=3) & Stdv & Avg. (n=3) & Stdv & \\
\hline NaFL in Water & 0.557 & 0.00239 & 7.93 & 0.0189 & 7.03 \\
\hline Post Coagulation & 0.527 & 0.00656 & 7.54 & 0.0796 & 6.99 \\
\hline Removal [\%] & \multicolumn{3}{|c|}{5.4} & \multicolumn{2}{c|}{4.9} \\
\hline
\end{tabular}



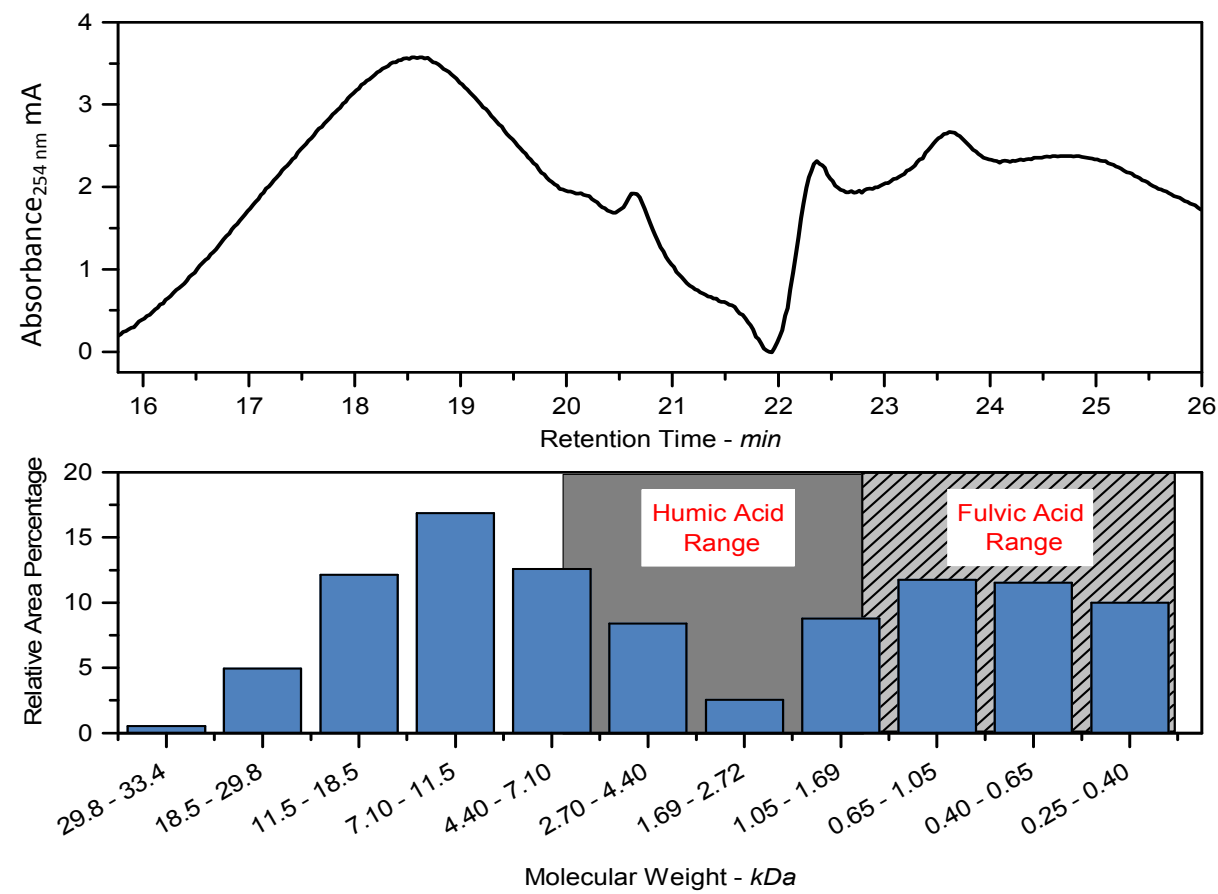

Fig. S2. Molecular weight profile of instant coffee mix used as "low SUVA" surrogate. Data obtained via HPLC-SEC. 


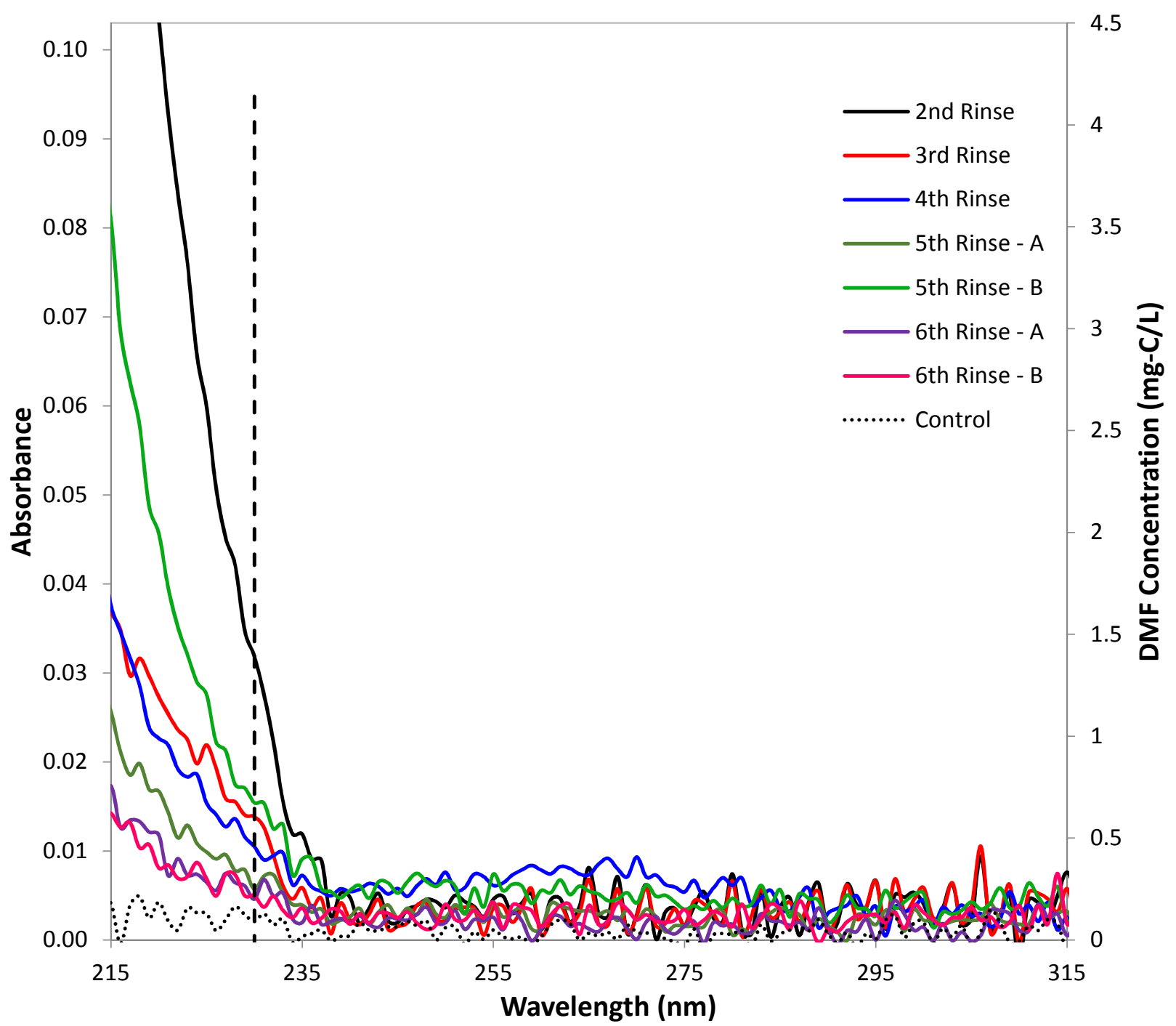

Fig. S3. Successive washes with water shows excess polymer is removed. After filtration the NanoResin is redispersed into $125 \mathrm{~mL}$ of water, then filtered and washed with $25 \mathrm{~mL}$ of water. The $25 \mathrm{~mL}$ filtrate is analyzed by UV spectroscopy. A calibration curve was measured to determine the extinction coefficient of DMF in water, where $\varepsilon_{230}=0.00414 \pm 0.00001(\mathrm{mg}-\mathrm{C} / \mathrm{L})^{-1} \cdot \mathrm{cm}^{-1}$, with a minimum detection of $<0.2 \mathrm{mg}-\mathrm{C} / \mathrm{L}$. After rinsing procedure, DMF was removed to below detection levels. 


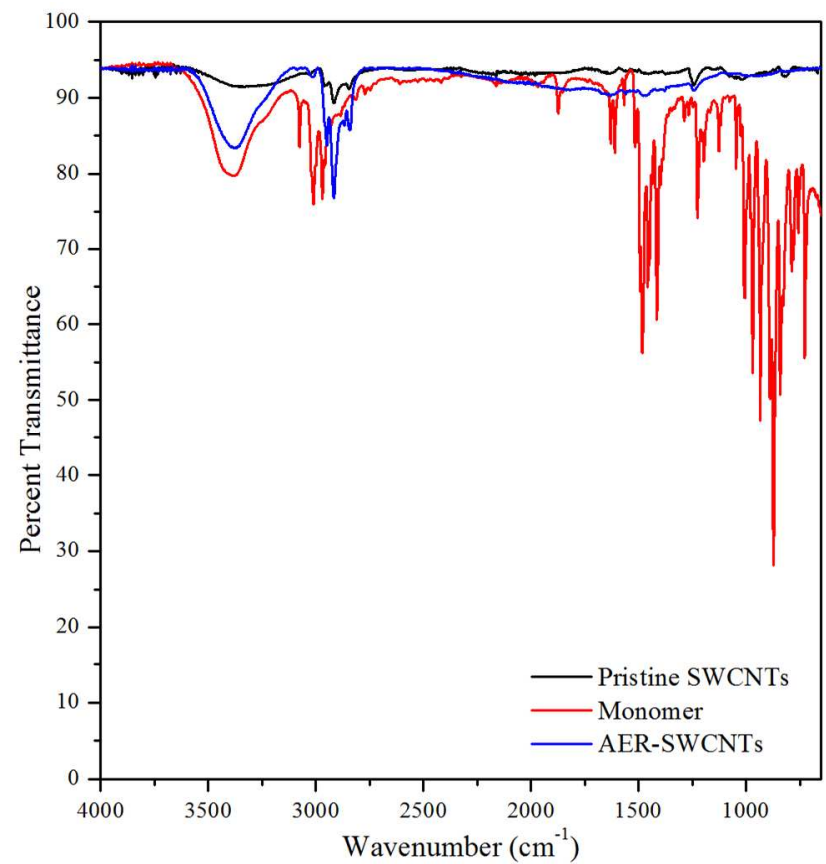

Fig. S4. Full FTIR overlay of monomer, pristine SWCNTs, and functionalized SWCNTs. 


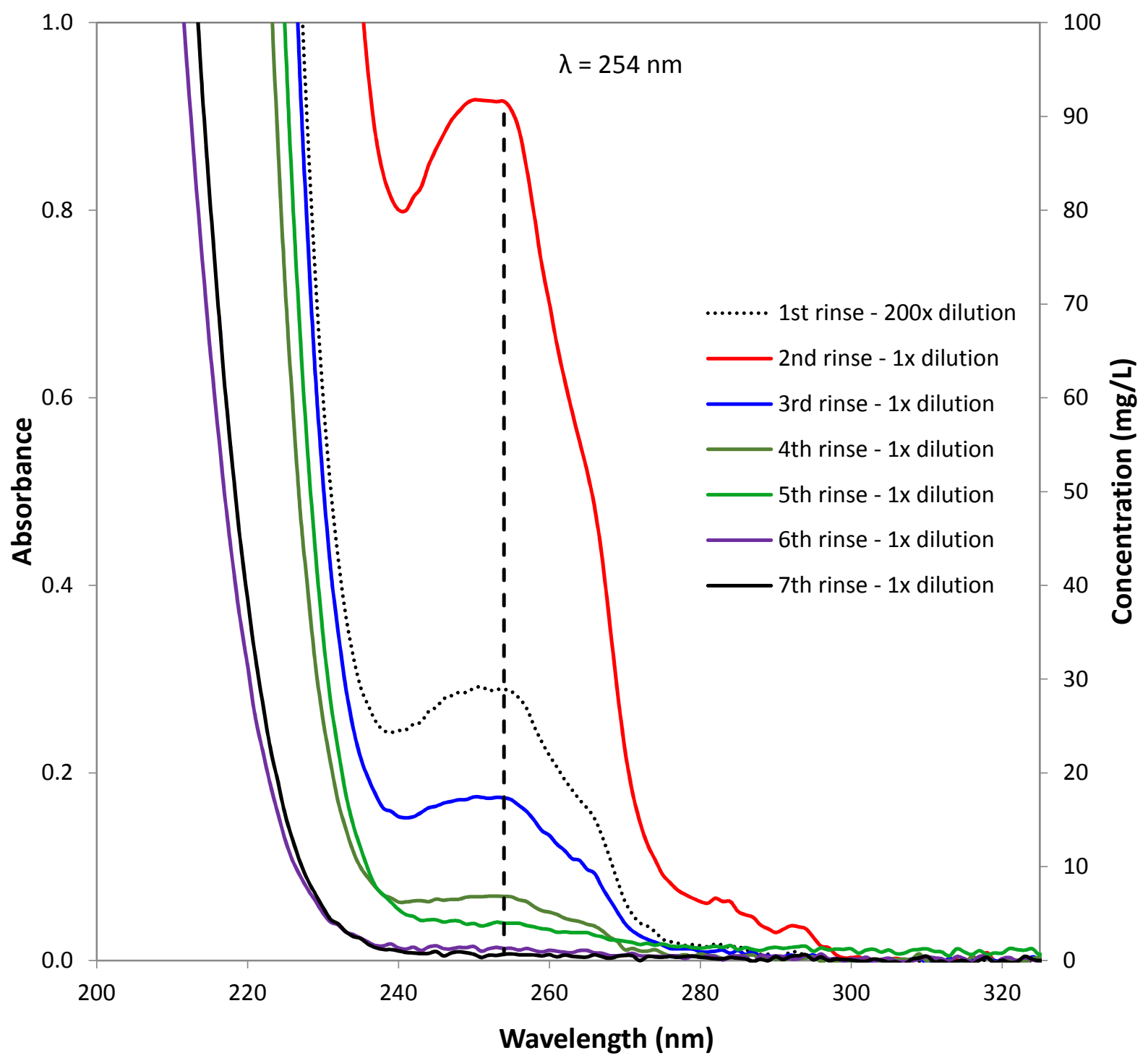

Fig. S5. Successive washes with $10 \mathrm{~mL}$ aliquots of water shows excess polymer is removed by rinsing with water. Note the spectrum of the first rinse (black dotted line) was taken after we diluted the filtrate 200x to get the absorbance $<1$. These spectra shows that most of the polymer is removed during the first rinse. After seven rinses, $<1 \mathrm{mg} / \mathrm{L}$ of polymer remained $\left(\varepsilon_{\text {poly-vbTMAC }}=0.0102 \pm 0.0007 \mathrm{M}^{-1} \cdot \mathrm{cm}^{-1}\right)$. The vertical dashed line is at $254 \mathrm{~nm}$ where the polymer concentration is measured and displayed on the right axis. 


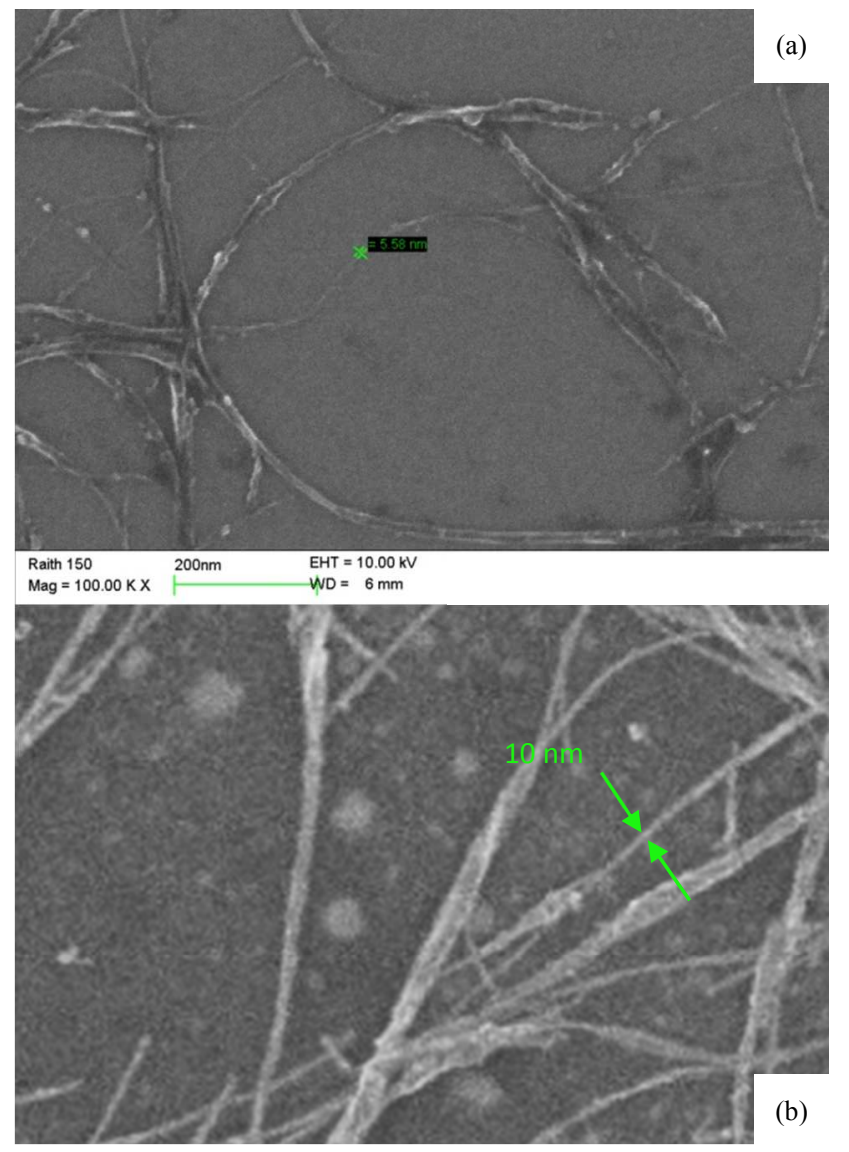

Fig. S6. (a) Scanning Electron Micrograph of pristine SWCNTs. Actual diameters are $<3 \mathrm{~nm}$, but appear larger due to electron beam broadening. (b)Scanning electron micrograph of AER coated SWCNTs showing a conformal polymer coating, as well as an increase in diameter to greater than $10 \mathrm{~nm}$. Magnification and scale bar are the same for both micrographs. 

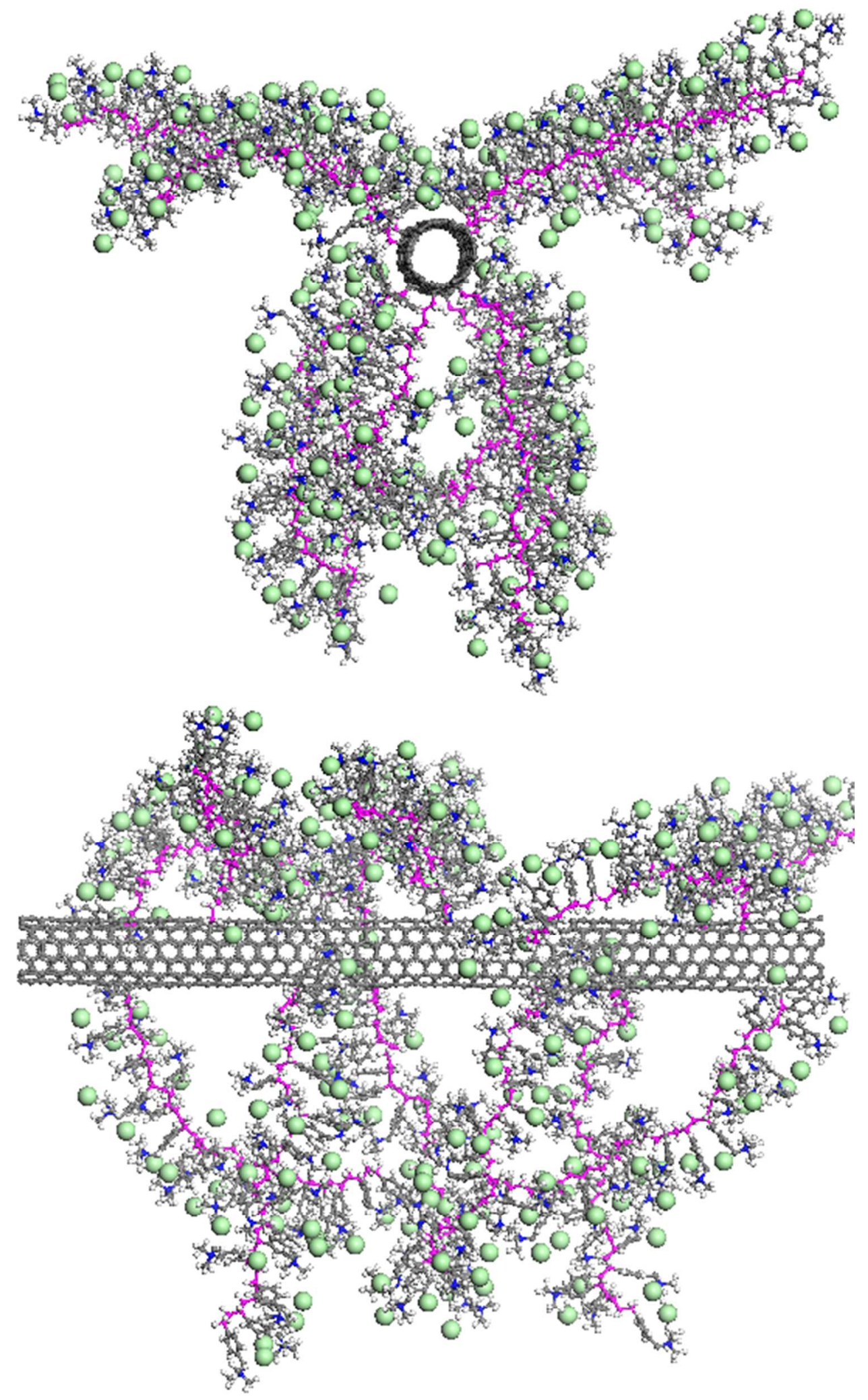

Fig. S7. Molecular dynamics anneal simulation snapshot of 12 poly(vbTMAC) strands (20 units long) covalently bonded to a $(6,6)$ SWCNT. Materials Studio 4.4 UFF after 5 annealing cycles 300 to $500 \mathrm{~K}$ and geometry optimization after each. Total simulation time $=100 \mathrm{ps}$ ( 2 fs steps), NVT. 


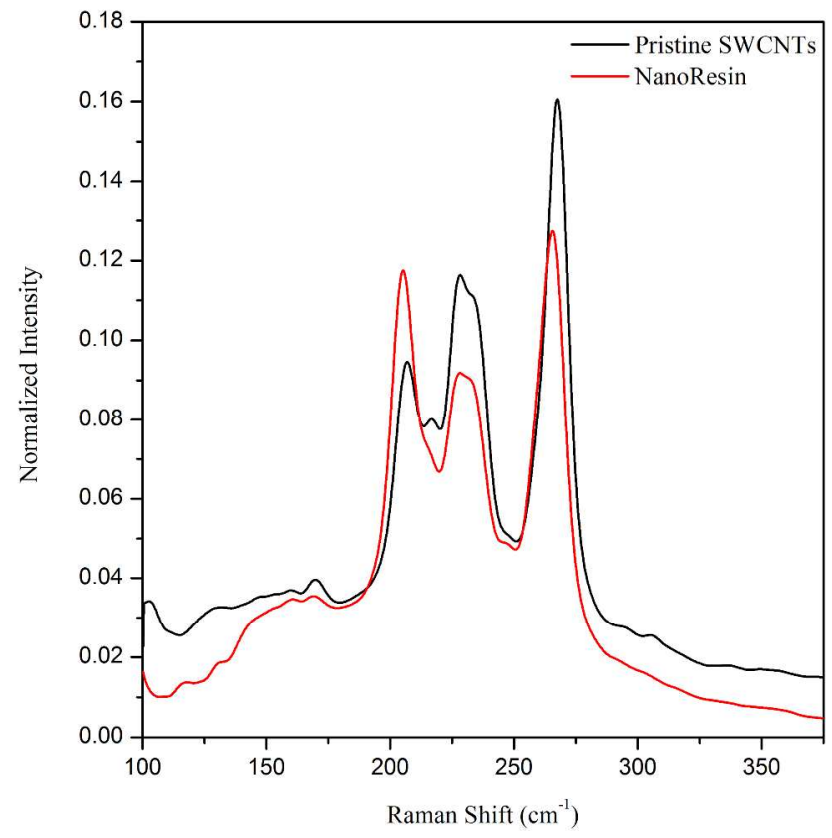

Fig. S8. Overlaid Raman spectra of pristine (black) and NanoResin (red) focusing on the Radial Breathing Modes of the tubes. Data shows the smaller diameter (higher energy Raman shift) tubes were more likely to be functionalized, compared to larger diameter tubes. 
Table S2. Raw data from Fig. 6, low MW surrogate (sodium fluorescein) adsorption after 10 second incubation.

\begin{tabular}{l|cccccc}
\hline \multicolumn{1}{c|}{$\begin{array}{c}\text { Adsorbent } \\
\text { Media }\end{array}$} & $\begin{array}{c}\text { Initial } \\
\text { Surrogate } \\
\text { Concentration } \\
(\mathbf{m g - C} / \mathbf{L})\end{array}$ & $\begin{array}{c}\text { Final } \\
\text { Surrogate } \\
\text { Concentration; } \\
\mathbf{C}_{\mathbf{f}}(\mathbf{m g - C} / \mathbf{L})\end{array}$ & $\begin{array}{c}\text { Surrogate } \\
\text { Volume } \\
(\mathbf{m L})\end{array}$ & $\begin{array}{c}\text { NaFL } \\
\text { removed } \\
(\mathbf{m g - C})\end{array}$ & $\begin{array}{c}\text { Mass of } \\
\text { Adsorbent } \\
\text { (mg) }\end{array}$ & $\begin{array}{c}\mathbf{q} \\
\text { (mg-C } \\
\mathbf{a d s o r b a t e} / \mathbf{g} \\
\mathbf{a d s o r b e n t})\end{array}$ \\
\hline NanoResin (FF) & 5.01 & 2.24 & 3.00 & $8.31 \times 10^{-3}$ & 0.17 & 50 \\
\hline NanoResin (inc.) & 6.70 & 5.03 & 1.75 & $2.91 \times 10^{-3}$ & 0.078 & 37 \\
\hline MIEX & 6.51 & 5.93 & 1.75 & $1.09 \times 10^{-3}$ & 0.18 & 6.2 \\
\hline Dowex & 6.51 & 6.49 & 1.75 & $8.55 \times 10^{-5}$ & 0.22 & 0.16 \\
\hline
\end{tabular}


Table S3. Raw data from Fig. 7, low molecular weight surrogate (sodium fluorescein) adsorption isotherm.

\begin{tabular}{|c|c|c|c|c|c|}
\hline $\begin{array}{l}\text { Adsorbent } \\
\text { Media }\end{array}$ & $\begin{array}{c}\text { Initial } \\
\text { Surrogate } \\
\text { Concentration } \\
(\mathrm{mg}-\mathrm{C} / \mathrm{L}) \\
\end{array}$ & $\begin{array}{c}\text { Final Surrogate } \\
\text { Concentration; } \\
c_{\text {eq }}(m g-C / L)\end{array}$ & $\begin{array}{c}\text { Surrogate } \\
\text { Volume (mL) }\end{array}$ & $\begin{array}{c}\text { Mass of } \\
\text { Adsorbent (mg) }\end{array}$ & $\begin{array}{c}\mathbf{q}_{\mathrm{e}} \\
\text { (mg adsorbate/ } \\
\text { g adsorbent) }\end{array}$ \\
\hline \multirow{5}{*}{ NanoResin } & 1.20 & 0.0915 & 15.0 & 0.18 & 92.7 \\
\hline & 2.04 & 0.636 & 15.0 & 0.18 & 116 \\
\hline & 3.05 & 1.50 & 15.0 & 0.18 & 127 \\
\hline & 3.91 & 2.53 & 15.0 & 0.17 & 119 \\
\hline & 6.10 & 4.59 & 15.0 & 0.17 & 126 \\
\hline \multirow{5}{*}{ MIEX } & 1.20 & 0.815 & 15.0 & 0.22 & 27.4 \\
\hline & 2.04 & 1.60 & 15.0 & 0.15 & 44.5 \\
\hline & 3.05 & 2.31 & 15.0 & 0.21 & 53.1 \\
\hline & 3.91 & 3.22 & 15.0 & 0.19 & 54.6 \\
\hline & 6.10 & 4.34 & 15.0 & 0.37 & 69.6 \\
\hline \multirow{5}{*}{ Dowex } & 1.20 & 1.12 & 15.0 & 0.27 & 5.26 \\
\hline & 2.04 & 1.79 & 15.0 & 0.26 & 14.7 \\
\hline & 3.05 & 2.68 & 15.0 & 0.24 & 23.3 \\
\hline & 3.91 & 3.45 & 15.0 & 0.24 & 28.9 \\
\hline & 6.10 & 5.24 & 15.0 & 0.35 & 34.9 \\
\hline
\end{tabular}




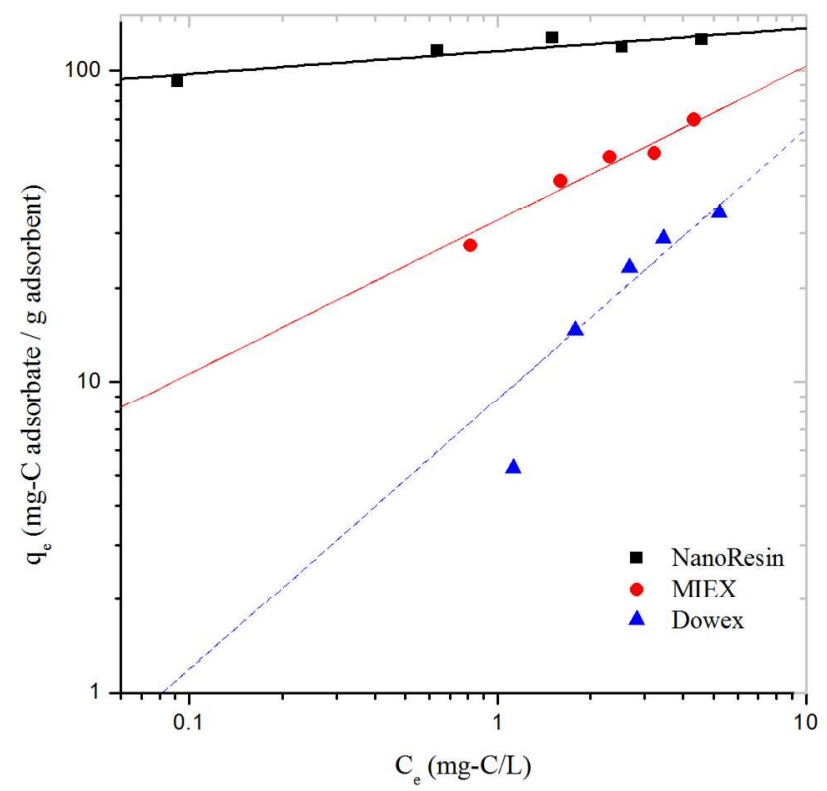

Fig. S9. Adsorption isotherm of low MW NaFL surrogate, plotted as a log-log graph, comparing commercially available adsorbents to NanoResin. All data are compared with a similar resin dose and adsorbent mass. As surface area increases (DOWEX® $21 \mathrm{~K}<$ MIEX ${ }^{\circledR}<$ NanoResin), more adsorbate can be bound per mass, much more efficiently. All samples were allowed to incubate for 15 hours. NanoResin and MIEX are at equilibrium within one hour. Solid line is from fitting the Freundlich isotherm to the data. Dashed line is a nonequilibrium fit to the Freundlich for DOWEX $21 \mathrm{~K}$. 
Table S4A. Raw data from 15 minute, low SUVA surrogate adsorption experiments.

*NanoResin film data collected by averaging five separate adsorption experiments, regenerating, and rinsing between each.

\begin{tabular}{c|cccccc}
\hline $\begin{array}{c}\text { Adsorbent } \\
\text { Media }\end{array}$ & $\begin{array}{c}\text { Mass of } \\
\text { Adsorbent } \\
\text { (mg) }\end{array}$ & $\begin{array}{c}\text { UV254 } \\
\text { initial }\end{array}$ & $\begin{array}{c}\text { UV254 } \\
\text { Removal } \\
\text { (initial - final) }\end{array}$ & $\begin{array}{c}\text { (UV removal) / } \\
\text { (g adsorbent) } \\
\text { (A.U./g) }\end{array}$ & $\begin{array}{c}\text { UV254 \% } \\
\text { difference }\end{array}$ & $\begin{array}{c}\text { (UV254 \% } \\
\text { difference) / (g } \\
\text { adsorbent) }\end{array}$ \\
\hline $\begin{array}{c}\text { NanoResin } \\
\text { Dispersion }\end{array}$ & 0.51 & 0.0447 & 0.0339 & 65.9 & 75.9 & $1.47 \mathrm{E} 5$ \\
\hline $\begin{array}{c}\text { NanoResin } \\
\text { Film* }\end{array}$ & 0.30 & 0.1481 & 0.0183 & 40.2 & 3.10 & 5.87 \\
\hline MIEX & 3.39 & 0.1789 & 0.0105 & 5.05 & 8.89 & $1.73 \mathrm{E} 3$ \\
\cline { 2 - 6 } & 3.15 & 0.1789 & 0.0159 & $2.82 \mathrm{E} 3$ \\
\hline
\end{tabular}

Table S4B. Raw data from 15 minute, low SUVA surrogate adsorption experiments.

*NanoResin film data collected by combining five separate adsorption experiments, regenerating, and rinsing between each.

\begin{tabular}{|c|c|c|c|c|c|c|}
\hline $\begin{array}{c}\text { Adsorbent } \\
\text { Media }\end{array}$ & $\begin{array}{c}\text { Mass of } \\
\text { Adsorbent } \\
\text { (mg) }\end{array}$ & $\begin{array}{c}\text { Initial } \\
\text { Surrogate } \\
\text { Concentration } \\
(\mathrm{mg}-\mathrm{C} / \mathrm{L})\end{array}$ & $\begin{array}{c}\text { Final } \\
\text { Surrogate } \\
\text { Concentration; } \\
\mathrm{C}_{\mathrm{eq}}(\mathrm{mg-C} / \mathrm{L})\end{array}$ & $\begin{array}{c}\text { TOC } \\
\% \text { difference }\end{array}$ & $\begin{array}{c}\text { (\% difference } \\
\text { TOC) / (g } \\
\text { adsorbent) }\end{array}$ & $\begin{array}{c}\mathrm{q}_{\mathrm{e}} \\
\text { (mg-C NOM) } \\
/ \\
\text { (g adsorbent) }\end{array}$ \\
\hline $\begin{array}{c}\text { NanoResin } \\
\text { Film* }\end{array}$ & 1.49 & 7.58 & 6.20 & 18.2 & 122.2 & 32.5 \\
\hline \multirow{2}{*}{ MIEX } & 3.39 & 8.60 & 8.03 & 6.63 & 19.6 & 6.19 \\
\hline & 3.15 & 8.60 & 7.71 & 10.3 & 32.8 & 9.56 \\
\hline
\end{tabular}


Table S5. Raw data from 15 minute, high SUVA surrogate adsorption experiments.

\begin{tabular}{c|cccccc}
\hline $\begin{array}{c}\text { Adsorbent } \\
\text { Media }\end{array}$ & $\begin{array}{c}\text { Mass of } \\
\text { Adsorbent } \\
\text { (mg) }\end{array}$ & $\begin{array}{c}\text { UV254 } \\
\text { initial }\end{array}$ & $\begin{array}{c}\text { UV254 } \\
\text { Removal } \\
\text { (initial - final) }\end{array}$ & $\begin{array}{c}\text { (UV removal) / } \\
\text { (g adsorbent) } \\
\text { (A.U./g) }\end{array}$ & $\begin{array}{c}\text { UV254 \% } \\
\text { difference }\end{array}$ & $\begin{array}{c}\text { (UV254 \% } \\
\text { difference) / } \\
\text { (g adsorbent) }\end{array}$ \\
\hline $\begin{array}{c}\text { NanoResin } \\
\text { Dispersion }\end{array}$ & 0.51 & 0.1452 & 0.1363 & 265 & 93.9 & $1.82 \mathrm{E} 5$ \\
\hline \multirow{2}{*}{ MIEX } & 0.51 & 0.1452 & 0.1368 & 266 & 94.2 & $1.83 \mathrm{E} 5$ \\
\hline & 2.55 & 0.4697 & 0.0109 & 4.27 & 2.32 & 910. \\
& 2.49 & 0.4697 & 0.0107 & 4.30 & 2.28 & 915 \\
\hline
\end{tabular}

Table S6A. Raw data for the low SUVA surrogate adsorption experiments. All samples were allowed to incubate for five minutes.

\begin{tabular}{c|cccccc}
\hline $\begin{array}{c}\text { Adsorbent } \\
\text { Media }\end{array}$ & $\begin{array}{c}\text { Mass of } \\
\text { Adsorbent } \\
\text { (mg) }\end{array}$ & $\begin{array}{c}\text { UV254 } \\
\text { initial }\end{array}$ & $\begin{array}{c}\text { UV254 Removal } \\
\text { (initial - final) }\end{array}$ & $\begin{array}{c}\text { (UV removal) } \\
\text { (g adsorbent) } \\
\text { (A.U./g) }\end{array}$ & $\begin{array}{c}\text { UV254 \% } \\
\text { difference }\end{array}$ & $\begin{array}{c}\text { (UV254 \% } \\
\text { difference) /(g } \\
\text { adsorbent) }\end{array}$ \\
\hline $\begin{array}{c}\text { NanoResin } \\
\text { Dispersion }\end{array}$ & 0.23 & 0.0817 & 0.0521 & 230. & 63.8 & $2.81 \mathrm{E} 5$ \\
\hline $\begin{array}{c}\text { NanoResin } \\
\text { Film }\end{array}$ & 0.35 & 0.188 & 0.0500 & 142 & 26.6 & $7.57 \mathrm{E} 4$ \\
\hline MIEX & 0.59 & 0.188 & 0.0140 & 23.7 & 7.45 & $1.26 \mathrm{E} 4$ \\
\hline
\end{tabular}

Table S6B. Raw data for the low SUVA surrogate adsorption experiments. All samples were allowed to incubate for five minutes.

\begin{tabular}{c|cccccc}
\hline $\begin{array}{c}\text { Adsorbent } \\
\text { Media }\end{array}$ & $\begin{array}{c}\text { Mass of } \\
\text { Adsorbent } \\
\text { (mg) }\end{array}$ & $\begin{array}{c}\text { Initial } \\
\text { Surrogate } \\
\text { Concentration } \\
\text { (mg-C/L) }\end{array}$ & $\begin{array}{c}\text { Final } \\
\text { Surrogate } \\
\text { Concentration; } \\
\mathbf{C}_{\text {eq }} \text { (mg-C/L) }\end{array}$ & $\begin{array}{c}\text { TOC } \\
\text { \% difference }\end{array}$ & $\begin{array}{c}\mathrm{q}_{\mathrm{e}} \\
\text { (\% difference } \\
\text { TOC) / (g } \\
\text { adsorbent) }\end{array}$ & $\begin{array}{c}\text { (mg-C NOM) } \\
/ \\
\text { (g adsorbent) }\end{array}$ \\
\hline $\begin{array}{c}\text { NanoResin } \\
\text { Film }\end{array}$ & 0.35 & 8.90 & 6.22 & 30.1 & 86000 & 53.4 \\
\hline MIEX & 0.59 & 8.90 & 6.99 & 21.5 & 36400 & 22.6 \\
\hline
\end{tabular}


Table S7A. Raw UV254 data from Section 3.7: Adsorption of Myrtle Beach, SC NOM concentrate onto NanoResin. Samples incubated for five minutes.

\begin{tabular}{c|cccccc}
\hline $\begin{array}{c}\text { Adsorbent } \\
\text { Media }\end{array}$ & $\begin{array}{c}\text { Mass of } \\
\text { Adsorbent } \\
\text { (mg) }\end{array}$ & $\begin{array}{c}\text { UV254 } \\
\text { initial }\end{array}$ & $\begin{array}{c}\text { UV254 Removal } \\
\text { (initial - final) }\end{array}$ & $\begin{array}{c}\text { (UV removal) / } \\
\text { (g adsorbent) } \\
\text { (A.U./g) }\end{array}$ & $\begin{array}{c}\text { UV254 \% } \\
\text { difference }\end{array}$ & $\begin{array}{c}\text { (UV254 \% } \\
\text { difference) /(g } \\
\text { adsorbent) }\end{array}$ \\
\hline $\begin{array}{c}\text { NanoResin } \\
\text { Film }\end{array}$ & 0.34 & 0.5026 & 0.1041 & 303 & 20.7 & $6.02 \mathrm{E} 4$ \\
\hline
\end{tabular}

Table. S7B. Raw TOC data from Section 3.7: Adsorption of Myrtle Beach, SC NOM concentrate onto NanoResin. Samples incubated for five minutes.

\begin{tabular}{c|cccccc}
\hline $\begin{array}{c}\text { Adsorbent } \\
\text { Media }\end{array}$ & $\begin{array}{c}\text { Mass of } \\
\text { Adsorbent } \\
(\mathbf{m g})\end{array}$ & $\begin{array}{c}\text { Initial } \\
\text { Surrogate } \\
\text { Concentration } \\
(\mathbf{m g - C} / \mathbf{L})\end{array}$ & $\begin{array}{c}\text { Final } \\
\text { Surrogate } \\
\text { Concentration; } \\
\mathbf{C}_{\text {eq }}(\mathbf{m g - C} / \mathbf{L})\end{array}$ & $\begin{array}{c}\text { TOC } \\
\% \text { difference }\end{array}$ & $\begin{array}{c}\mathbf{q}_{\mathrm{e}} \\
\text { (\% difference } \\
\text { TOC } /(\mathbf{g} \\
\text { adsorbent) }\end{array}$ & $\begin{array}{c}\text { (mg-C NOM) } \\
/ \\
\text { (g adsorbent) }\end{array}$ \\
\hline $\begin{array}{c}\text { NanoResin } \\
\text { Film }\end{array}$ & 0.34 & 11.5 & 9.17 & 20.4 & $5.93 \mathrm{E} 4$ & 235 \\
\hline
\end{tabular}




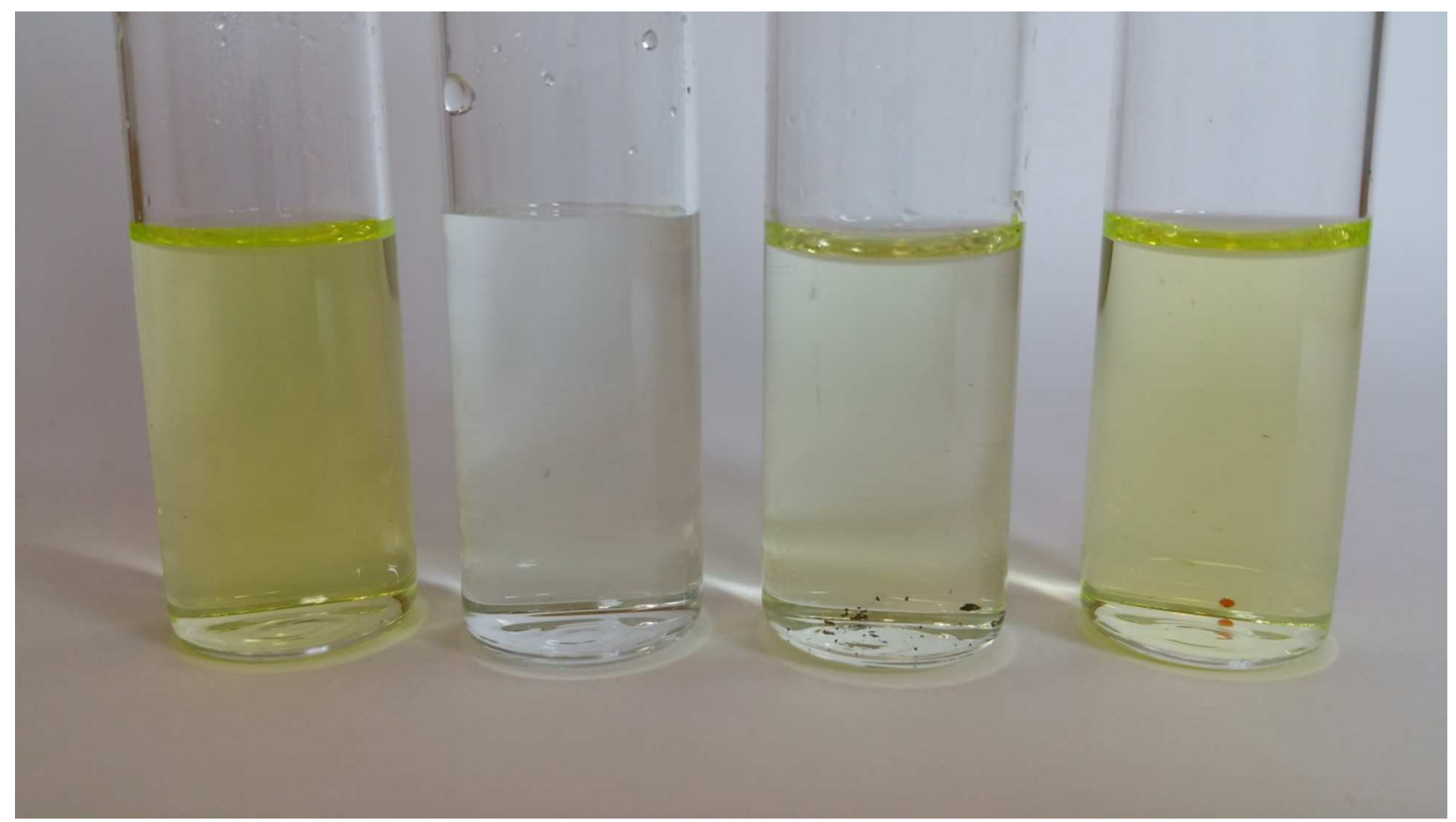

Fig. S10: Photo of NaFL removal is with the $1.2 \mathrm{mg}-\mathrm{C} / \mathrm{L}$ control sample on the left, followed by the NanoResin dispersion, MIEX, and Dowex after incubating on a tube rotator for 15 hours.
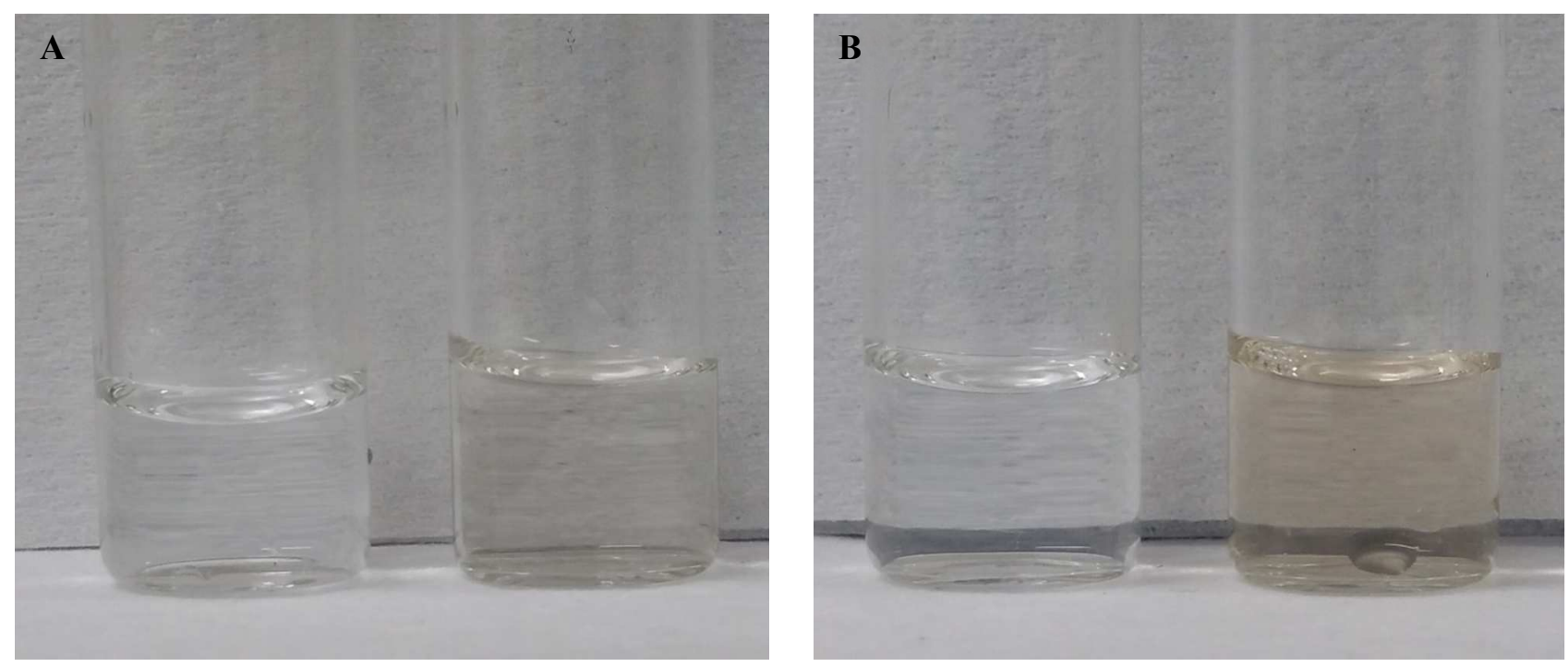

Fig. S11: 15 minute incubation of NanoResin dispersion with low SUVA $7.58 \mathrm{mg}-\mathrm{C} / \mathrm{L}$ (A) and high SUVA (B). Initial concentration of low SUVA sample in (A) is $8.60 \mathrm{mg}-\mathrm{C} / \mathrm{L}$ (right). Incubated sample on the (left). Initial concentration of high SUVA sample in (B) is $11.28 \mathrm{mg}-\mathrm{C} / \mathrm{L}$ (right). Incubated sample on the (left). 